\title{
Treatment of fungal pyelonephritis and ureterolithiasis with a subcutaneous ureteral bypass system and systemic antifungal medication in a cat
}

\author{
Rebecca G McQuitty' \\ Erinne $M$ Branter ${ }^{2}$ \\ 'Emergency and Critical Care \\ Department, ACCESS Specialty Animal \\ Hospital, Culver City, CA, USA; \\ ${ }^{2}$ Interventional Radiology/Endoscopy \\ Department, ACCESS Specialty Animal \\ Hospital, Culver City, CA, USA
}

This article was published in the following Dove Press journal: Veterinary Medicine: Research and Reports

\begin{abstract}
Purpose: We describe a case of ureteral obstruction and fungal pyelonephritis in a cat. The case was managed successfully with a subcutaneous ureteral bypass (SUB) device and systemic antifungal medication.
\end{abstract}

Case summary: A 9-year-old, male, neutered, domestic shorthair cat was referred for severe azotemia, ureteral obstruction, and sonographically identified progressive pyelectasia of the left kidney. A SUB device was placed. Cytology of the left kidney subsequently identified fungal organisms, and the cat responded clinically to treatment with fluconazole and supportive care. The cat survived for 11 months with intermittent fluconazole treatment.

Conclusion: Fungal pyelonephritis should be considered as a possible concurrent diagnosis in cats with ureteral obstruction. The successful clinical outcome in this case suggests that treatment with SUB placement, antifungal medication, and supportive care could be an effective treatment strategy.

Keywords: interventional radiology, ureteral obstruction, mycoses, invasive fungal disease, urinary tract infection

\section{Case report}

A 9-year-old, male, neutered, domestic shorthair cat was presented to his primary veterinarian for lethargy and decreased appetite. Nine months prior to this presentation, the patient had been diagnosed with renal disease; at that time blood urea nitrogen (BUN) had been $107 \mathrm{mg} / \mathrm{dL}$ (reference interval [RI] 15-34 mg/dL), creatinine $9.2 \mathrm{mg} /$ $\mathrm{dL}$ (RI 0.8-2.3 mg/dL), and urine specific gravity (USG) 1.019. Uroliths had been noted in the record, although no diagnostic imaging reports are available. Following fluid therapy and other supportive care, renal values had improved by the following month to BUN $32 \mathrm{mg} / \mathrm{dL}$ and creatinine $2.1 \mathrm{mg} / \mathrm{dL}$.

Now on presentation to the primary veterinarian, severe azotemia had recurred with BUN 96 (RI 16-36) and creatinine 11.3 (RI 0.8-2.4 mg/dL). Complete blood count was consistent with a stress leukogram: white blood cells $14 \times 10^{9} / \mathrm{L}$ (RI $5.5 \times 10^{9}-19.5 \times 10^{9} / \mathrm{L}$ ), segmented neutrophils $76 \%$ (RI 35\%-75\%), and lymphocytes 14\% (RI 20\%-45\%). Urinalysis identified isosthenuria (USG 1.012), with a normal protein:creatinine ratio of 0.3 . No urine culture was performed. An ELISA test was negative for feline immunodeficiency virus antibody, feline leukemia virus antigen, and heartworm antigen. Treatment included fluid therapy and cefovecin $8 \mathrm{mg} / \mathrm{kg}$
Correspondence: Rebecca G McQuitty Emergency and Critical Care Department, Silicon Valley Veterinary Specialists, 7160 Santa Teresa Boulevard, San Jose, CA 95139, USA

Tel +l 4086497070

Fax +I 4086497072

Email rebecca.mcquitty@svvspets.net 
subcutaneously (SC) (Convenia; Zoetis Inc, Kalamazoo, MI, USA), but on the seventh day of treatment azotemia had worsened, with BUN $201 \mathrm{mg} / \mathrm{dL}$ and creatinine $16.2 \mathrm{mg} / \mathrm{dL}$. Abdominal radiographs showed a small right kidney, mildly enlarged left kidney, punctate mineral opacity in the pelvic region of the left kidney, and multiple mineral opacities suggestive of ureteral calculi. The patient was then referred to a specialty hospital (Hospital A).

On presentation to Hospital A, a grade II/VI systolic heart murmur was ausculted, and Doppler blood pressure was $160 \mathrm{mmHg}$. Urine culture was submitted and was negative. Ultrasonography of the abdomen showed bilateral dilation of the renal pelves (right $0.3 \mathrm{~cm}$, left $0.45 \mathrm{~cm}$ ), left renoliths, left ureteral dilation with ureterolithiasis, decreased corticomedullary distinction in the kidneys bilaterally, and a right kidney smaller than the left (length in transverse section: right $2.05 \mathrm{~cm}$, left $4.23 \mathrm{~cm}$ ). Whole body radiographs showed minimal left atrial enlargement and an enlarged, irregular left kidney ( $6 \mathrm{~cm}$ in length) with left-sided nephrolithiasis and ureterolithiasis. Medical therapy was instituted with continued fluid diuresis, amlodipine $0.15 \mathrm{mg} / \mathrm{kg}$ by mouth (PO) q24h, ampicillin/sulbactam $22 \mathrm{mg} / \mathrm{kg}$ intravenously (IV) q12h, amitriptyline $1.2 \mathrm{mg} / \mathrm{kg}$ PO q24h, prazosin $0.06 \mathrm{mg} /$ $\mathrm{kg}$ PO q12h, famotidine $0.48 \mathrm{mg} / \mathrm{kg}$ IV q24h, buprenorphine $0.007 \mathrm{mg} / \mathrm{kg} \mathrm{SC} \mathrm{q} 12 \mathrm{~h}$, and aluminum hydroxide. After 2 days BUN improved slightly to $179 \mathrm{mg} / \mathrm{dL}$ (RI 7-27 mg/dL), but creatinine progressively increased to $18.5 \mathrm{mg} / \mathrm{dL}$ (RI $0.5-1.8$ $\mathrm{mg} / \mathrm{dL})$. Blood pressure increased to $190 \mathrm{mmHg}$ measured via Doppler. Repeat sonography revealed progressive dilation of the left renal pelvis to $0.57 \mathrm{~cm}$ (Figure 1), while

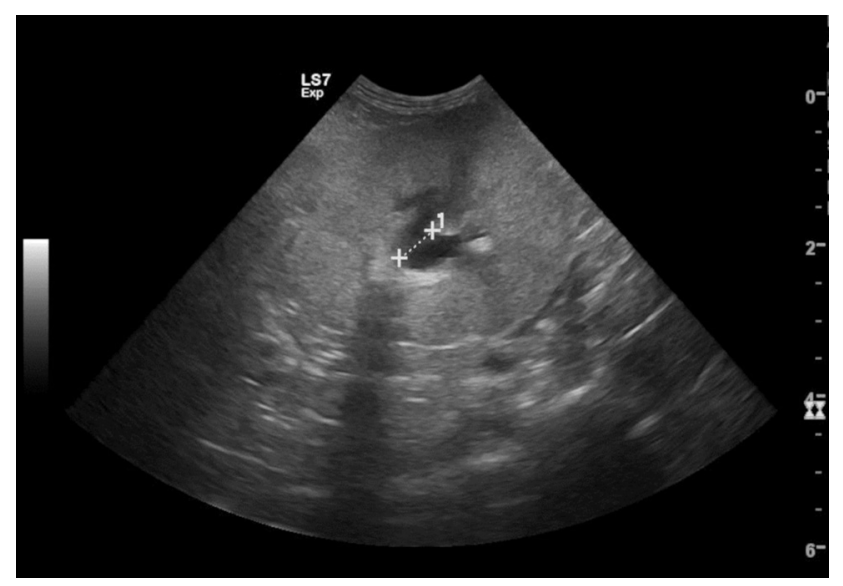

Figure I Transverse ultrasonographic image of the left kidney prior to surgery. Notes: Sonogram of the left kidney of a cat referred for progressive azotemia and progressive pyelectasia of the left kidney. White hash marks demarcate the borders of a dilated renal pelvis (width $0.57 \mathrm{~cm}$ ). Depth is marked in centimeters on the right of the image.

Abbreviation: LS7 Exp, Logiq S7 Expert. the right renal pelvis remained stable. Repeat radiographs showed evidence of fluid overload with a mild increase in the size of the cardiac silhouette and prominent caudal lobar pulmonary vasculature. There were wispy fluid opacities in the retroperitoneal space (Figure 2).

The cat was then referred to a second specialty hospital (Hospital B). On arrival at Hospital B, the patient was tense on palpation of the left kidney and had a grade II/VI heart murmur. He appeared well hydrated, and his mucous membranes, body condition score, and lung sounds were normal. Baseline in-house labwork showed BUN $>140 \mathrm{mg} / \mathrm{dL}$ (RI 15-34 mg/dL), creatinine $14.9 \mathrm{mg} / \mathrm{dL}$ (RI 1.0-2.2 mg/dL), and potassium $3.9 \mathrm{mmol} / \mathrm{L}$ (RI 2.9-4.2 $\mathrm{mmol} / \mathrm{L}$ ). Because the patient was presented during emergency hours, echocardiogram was deferred until the following day.

Given concern for ureteral obstruction, decompression of the left kidney via subcutaneous ureteral bypass (SUB) or double pigtail ureteral stent was planned. Surgical approach to the abdomen revealed a moderate amount of free abdominal fluid. The left kidney was enlarged and pale in color with vascularization of the renal capsule. The right kidney was small and irregular. In light of the abnormal gross appearance of the left kidney, an aspirate of the left renal cortex for cytology was performed (22 gauge needle, $3 \mathrm{~mL}$ syringe). In addition, urine was collected from the renal pelvis for bacterial and fungal culture using a 22 gauge IV catheter. Intraoperative fluoroscopic pyelogram demonstrated a tortuous, dilated proximal ureter with a proximal obstruction. Placement of a ureteral stent was attempted and was discontinued when a $150 \mathrm{~cm} \times 0.018 \mathrm{inch}$ guide wire (Weasel Wire; Infiniti Medical LLC, Redwood

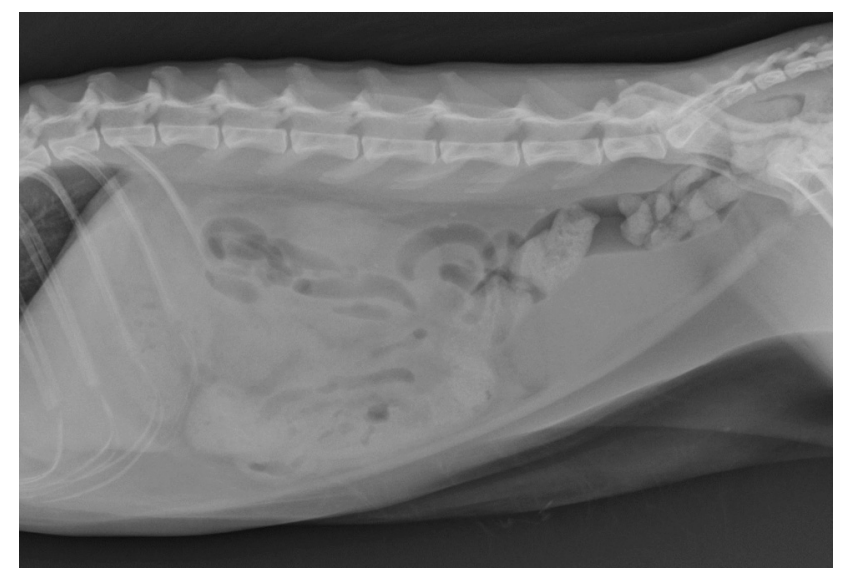

Figure 2 Right lateral radiographic projection of the abdomen prior to surgery. Notes: One large and one small kidney are apparent in this preoperative lateral radiograph. There are small mineral-dense opacities in the region of the ureters as well as a wispy opacity in the retroperitoneal region. 
City, CA, USA) would not feed past the ureterovesicular junction. A SUB device (Norfolk Vet Products, Skokie, IL, USA), consisting of a nephrostomy tube and a cystostomy tube connected by a subcutaneous infusion port, was then placed as described elsewhere. ${ }^{1}$ A contrast study confirmed the patency of the system. A Jackson-Pratt drain (MILA International, Florence, KY, USA), 3.5 French red rubber urinary catheter (Covidien LLC, Mansfield, MA, USA), 14 French esophagostomy tube (MILA International) in the left mid-cervical region, and 5.5 French $\times 13 \mathrm{~cm}$ triple-lumen catheter (Jorgensen Labs, Loveland, CO, USA) in the right jugular vein were placed. Prior to recovery, a right lateral radiographic projection was obtained to document proper placement of the SUB system (Figure 3).

Immediate postoperative supportive care consisted of a combination of a balanced isotonic crystalloid (Plasmalyte A; Baxter International Inc, Deerfield, IL, USA) and a hypotonic crystalloid (2.5\% dextrose and $0.45 \%$ sodium chloride; Abbot Laboratories, North Chicago, IL, USA), ticarcillin/clavulanic acid (GlaxoSmithKline, Research Triangle Park, NC, USA) $50 \mathrm{mg} / \mathrm{kg}$ IV q8h, gastroprotectants, and analgesia.

On postoperative day 1 , echocardiogram found left ventricular concentric hypertrophy (left ventricular posterior wall at end diastole $6.59 \mathrm{~mm}$, interventricular septum at end diastole $6.71 \mathrm{~mm}$ ) consistent with either chronic hypertension or primary hypertrophic cardiomyopathy. Evidence of fluid overload included mild pericardial effusion and mild dilation

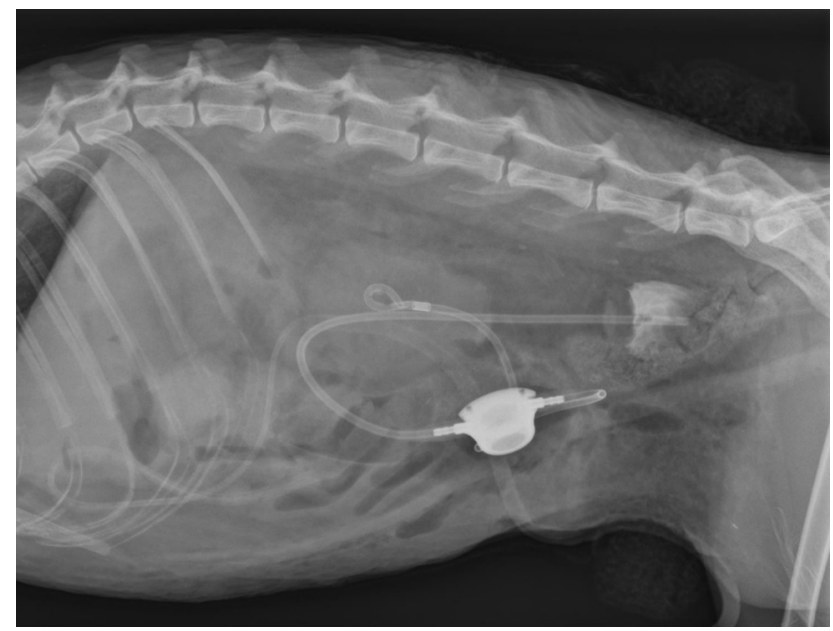

Figure 3 Right lateral radiographic projection of the abdomen immediately following surgery.

Notes: This postoperative lateral radiograph shows the SUB system (Norfolk Vet Products, Skokie, IL, USA), including nephrostomy tube, subcutaneous port, and cystoscopy tube. A Jackson-Pratt drain (MILA International, Florence, KY, USA) is also visible. Radiographic contrast (omnipaque; GE Healthcare, Princeton, NJ, USA) remains within the urinary bladder.

Abbreviation: SUB, subcutaneous ureteral bypass. of all chambers. IV fluid therapy was then minimized, with additional water provided via esophagostomy tube.

On postoperative day 2 , the urinary catheter was removed. The patient's renal values initially improved rapidly, with a blood gas on postoperative day 3 showing BUN $65 \mathrm{mg} / \mathrm{dL}$ (RI 15-34 mg/dL) and creatinine $2.4 \mathrm{mg} / \mathrm{dL}$ (RI 1.0-2.2 mg/ $\mathrm{dL}$ ). Ticarcillin/clavulanic acid was discontinued in favor of amoxicillin/clavulanate (Clavamox; Zoetis Inc) $12.9 \mathrm{mg} / \mathrm{kg}$ PO q12h.

Four days postoperatively, repeat sonography of the left kidney showed that pyelectasia had resolved (Figure 4). The patient experienced a urethral obstruction, which was relieved with a 3.5 French red rubber urinary catheter, yet on the following day BUN increased to $>140 \mathrm{mg} / \mathrm{dL}$ (RI 15-34) and creatinine to $9.3 \mathrm{mg} / \mathrm{dL}$ (RI $1.0-2.2 \mathrm{mg} / \mathrm{dL}$ ). Alanine aminotransferase became elevated (137 U/L; RI 12-130 U/L). The cat developed relative oliguria (urinary output $0.68 \mathrm{~mL} / \mathrm{kg} / \mathrm{hr}$ ) and had evidence of continuing fluid overload as indicated by generalized subcutaneous edema and chemosis. Furosemide (Vedco Inc, St Joseph, MD, USA) $1.9 \mathrm{mg} / \mathrm{kg}$ IV was given twice, followed by a constant rate infusion of furosemide $0.25 \mathrm{mg} / \mathrm{kg} / \mathrm{hr}$. Enrofloxacin (Baytril; Bayer HealthCare LLC, Shawnee Mission, KS, USA) $5 \mathrm{mg} / \mathrm{kg}$ IV q24h was added, and amoxicillin/clavulanate was discontinued. The patient inadvertently removed his urethral catheter and was able to urinate productively.

Six days postoperatively, renal cytology obtained at the time of surgery became available. Slides were cellular and considered to be of excellent quality. A moderate mixed

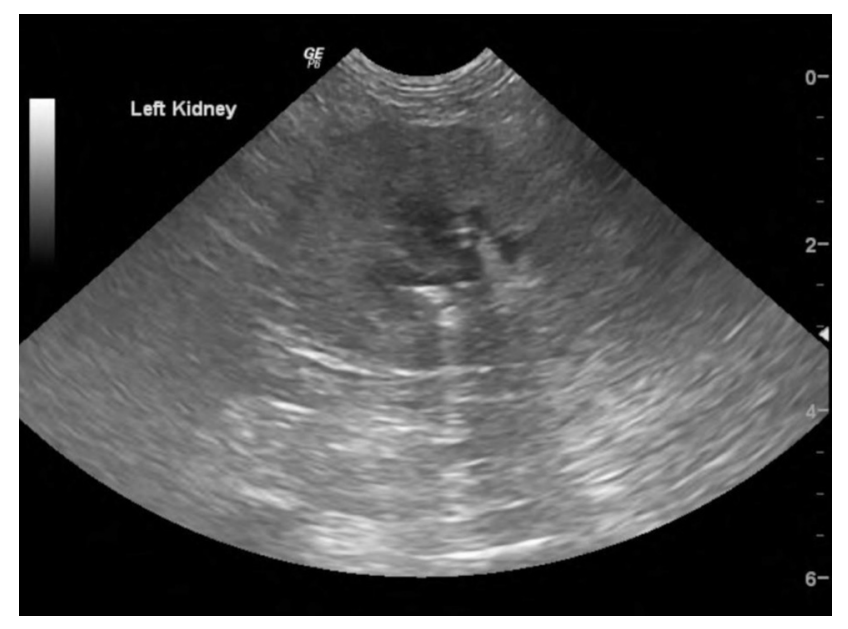

Figure 4 Transverse ultrasonographic image of the left kidney 4 days following surgery.

Notes: Pyelectasia has resolved 4 days following placement of a SUB device. Depth is marked in centimeters on the right of the image.

Abbreviations: GE P6, General Electric Logiq P6; SUB, subcutaneous ureteral bypass. 
inflammation of neutrophils, macrophages, and rare eosinophils was present. Oval and occasional budding fungal/yeast organisms were identified free throughout the background and phagocytized by macrophages; the appearance of the organisms was consistent with Candida. Bacterial and fungal cultures of urine aspirated from the renal pelvis at the time of surgery were negative, and a concentrated cytocentrifuge urine preparation for cytology was only sparsely cellular with no infectious organisms observed. Treatment for fungal infection was started with fluconazole oral suspension (Greenstone LLC, Peapack, NJ, USA) $7 \mathrm{mg} / \mathrm{kg}$ enterally q12h. Enrofloxacin was discontinued.

On the same day, the port of the SUB system obstructed with debris and was exchanged under anesthesia. On postoperative day 7 , the patient's packed cell volume decreased to $15 \%$ with total protein $6.2 \mathrm{~g} / \mathrm{dL}$. A transfusion of packed red blood cells $(35 \mathrm{~mL})$ was administered without complication following doses of furosemide $2 \mathrm{mg} / \mathrm{kg}$ IV and dexamethasone SP (Bimeda-MTC Animal Health Inc, Cambridge, Ontario, CA) $0.1 \mathrm{mg} / \mathrm{kg}$ IV.

On postoperative day 8 , creatinine and potassium continued to rise, and the patient showed evidence of progressive fluid overload, now including the development of pleural effusion. Thoracocentesis yielded $110 \mathrm{~mL}$ of pleural fluid.

Kidney values peaked postoperatively on day 9 (3 days after starting fluconazole), with BUN >140 mg/dL (RI 15-34 $\mathrm{mg} / \mathrm{dL}$ ) and creatinine $11.6 \mathrm{mg} / \mathrm{dL}$ (RI 1.0-2.2 mg/dL). Following this time azotemia improved rapidly; on day 13 , BUN was $56 \mathrm{mg} / \mathrm{dL}$ and creatinine $2.8 \mathrm{mg} / \mathrm{dL}$ (Figure 5). Ultrasound showed scant perirenal fluid on the left. The SUB

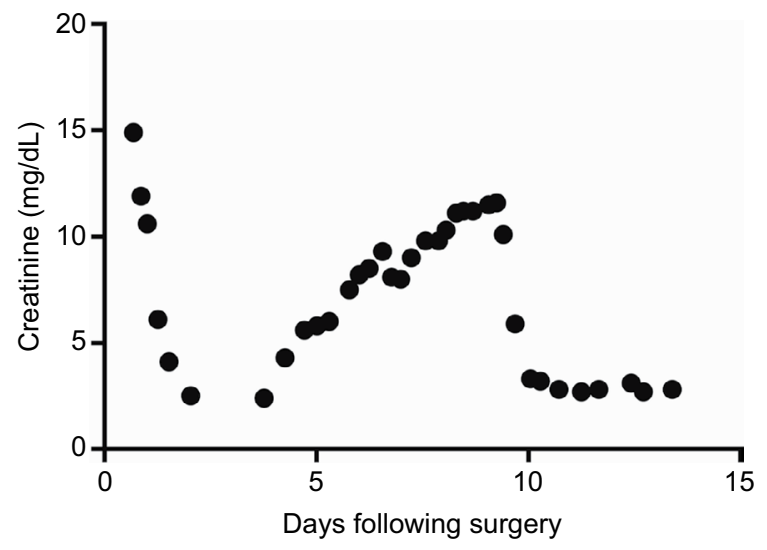

Figure 5 Creatinine values obtained in-house during hospitalization, plotted against time following placement of a SUB device.

Notes: Azotemia improved rapidly following SUB placement on day 0. A urethral obstruction was relieved on day 4. On day 6, treatment with fluconazole was started, and the SUB port was exchanged due to obstruction. Medical therapy was continued, and azotemia again showed rapid improvement on day 9.

Abbreviation: SUB, subcutaneous ureteral bypass. system was flushed, demonstrating normal flow. The patient was discharged home, and fluconazole was continued for 3 months.

Twelve weeks postoperatively, the patient experienced clinical signs of hyporexia and vocalization while eating, with a feline pancreatic lipase (Spec fPL; IDEXX Laboratories, Westbrook, ME, USA) of $32 \mu \mathrm{g} / \mathrm{L}$ (RI 0-3.5 $\mu \mathrm{g} / \mathrm{L}$ ). There was no obstruction of the SUB or urethra, and no renal pelvic dilation when examined sonographically. A urine culture at the same time grew Enterococcus $>100,000$ organisms $/ \mathrm{mL}$. The patient was successfully treated for presumptive pancreatitis and the urinary tract infection. Five months postoperatively, the patient was doing well with creatinine $2.5 \mathrm{mg} / \mathrm{dL}$ and BUN $39 \mathrm{mg} / \mathrm{dL}$; bacterial and fungal cultures of urine were negative. Seven months postoperatively, the patient's renal values increased again (creatinine $5.6 \mathrm{mg} / \mathrm{dL}$, BUN $70 \mathrm{mg}$ / dL). His SUB system remained patent, and bacterial urine culture was negative; fungal urine culture was not repeated. Fluconazole was resumed (6.8 mg/kg PO q12h), and 1 week thereafter renal values again decreased (creatinine $3.1 \mathrm{mg} / \mathrm{dL}$, BUN $37 \mathrm{mg} / \mathrm{dL}$ ). Eleven months (338 days) postoperatively, the patient was euthanized due to progression of his kidney disease despite systemic antifungal therapy and a patent SUB system. Postmortem histopathology of the kidney showed chronic interstitial fibrosis with tubulointerstitial nephritis, glomerulonephritis, and tubular dilation; these changes are consistent with chronic pyelonephritis and obstructive disease. No organisms were found on Grocott/Gimori's methenamine silver stain, and fungal culture of renal tissue was negative.

\section{Discussion}

The present case describes the treatment of fungal pyelonephritis and ureteral obstruction with placement of a SUB device and systemic treatment with fluconazole. To our knowledge, ureteral obstruction with a fungal organism present and resolution of signs with decompression and antifungal therapy has not previously been reported. Multiple complications were successfully addressed during the postoperative period including urethral obstruction, obstruction of the SUB port, anemia, and fluid overload.

Fungal urinary tract infections in cats are uncommon and are usually associated with predisposing conditions including diabetes mellitus, renal failure, and lower urinary tract diseases. ${ }^{2,3}$ The most commonly reported fungal organisms in feline urine cultures are Candida species. ${ }^{3}$ Fungal pyelonephritis has been reported both as a localized infection and as a manifestation of systemic fungal disease. ${ }^{4-6}$ Urolithiasis has 
been reported in both cats and dogs with fungal urinary tract infections. ${ }^{2,7}$ Ureteral calculi are the most common cause of feline ureteral obstruction, while less common causes include strictures, dried solidified blood clots, and neoplasia. ${ }^{1,8}$ In general, the reported incidence of ureterolithiasis in cats has increased in recent decades. ${ }^{8,9}$ Options for treatment of ureteral obstructions are indwelling double pigtail ureteral stent placement, the SUB device, ureterotomy, ureteroneocystostomy, or as a last resort, nephroureterectomy. ${ }^{1,10-14} \mathrm{~A}$ recent case series described treatment with a SUB device in four cats with bacterial pyonephrosis and ureteral obstruction. ${ }^{15}$

Fungal pyelonephritis was diagnosed in this case based on fungal organisms on renal cytology, including intracellular organisms, and on the abnormal gross appearance of the kidney during surgery. Moreover, there was significant improvement in azotemia following therapy with fluconazole, where simple relief of the ureteral obstruction was insufficient. When azotemia progressed 7 months post-intervention, renal values again responded favorably to fluconazole. These factors mitigate against the possibility that fungal organisms found on cytology were the result of contamination. No other infectious organism or neoplasia was identified on renal cytology. Both bacterial and fungal urine cultures as well as cytology of a cytocentrifuge preparation of urine from the renal pelvis were negative, although most Candida species can grow on standard urine bacterial culture. ${ }^{16}$ To our knowledge, no published reports have indicated the sensitivity of culture compared with cytology or histopathology for mycotic infection in the small animal urinary tract. In humans, criteria for diagnosis of invasive fungal infections are available only for research purposes. ${ }^{17,18}$ According to those criteria, cytopathologic examination of a sample from a sterile site showing yeast cells is classified as a proven infection, although confirmation with culture would provide further evidence. A PCR assay targeting fungal sequences in urine or tissue can be considered for fungal identification in the future. ${ }^{19}$

Obstruction of the left ureter required emergency therapy. The small size of the right kidney and severity of azotemia ruled out nephrectomy as an option. After placement of a left ureteral stent proved impossible, therapeutic options included placement of the SUB device or ureterotomy; recent retrospective studies have shown good long-term survival and decreased risk of recurrent ureteral obstruction with SUB placement ${ }^{12}$ compared with traditional surgery. ${ }^{10,20-22}$ Fluconazole was chosen for antifungal therapy based on its urinary excretion and its relative safety compared to other antifungal agents. The addition of amphotericin B was considered if the patient had not responded to fluconazole alone, but was suboptimal due to its nephrotoxic potential.

Use of antibacterial therapy in this case can be questioned given negative culture results, normothermia, and lack of significant neutrophilia or band neutrophils. In cats with ureteral calculi, bacterial urinary tract infections have been documented in $33 \%$ of cats in one study and $8 \%$ in another study. ${ }^{1,8}$ This patient received cefovecin prior to any urine culture, increasing the likelihood that the negative urine culture may have been inaccurate. Thus, a surgical implant was placed within a potentially infected organ system. Ticarcillin/ clavulanic acid was initially selected for its broad spectrum and urinary excretion. Initial treatment did not include enrofloxacin due to the risk of retinal toxicosis in cats; however, enrofloxacin is the first choice for pyelonephritis recommended by consensus guidelines, ${ }^{23}$ and it was chosen as the patient's condition continued to progress. No retinal toxicosis occurred in this patient. Treatment for potential bacterial pyelonephritis was discontinued once fungal infection was identified on cytology.

A recent review of cats treated with SUB devices found a median survival time (MST) of 272 days in cats with International Renal Interest Society (IRIS) stage 3 and 4 chronic kidney disease; more than half of the cats at earlier stages were alive at the time of the study and so MST was not reached. ${ }^{12}$ Survival for this patient was comparable to the cats with IRIS stage 3 and 4 in this prior study. In a case series of four cats with bacterial pyonephrosis and ureteral obstruction treated with SUB placement, median survival had not been reached but was over 559 days. ${ }^{15}$

\section{Conclusion}

The present study appears to be the first to document treatment of ureteral obstruction and concurrent fungal pyelonephritis with ureteral bypass and antifungal therapy. The patient was successfully treated with placement of a SUB system and systemic therapy with fluconazole. Survival in this patient was comparable to other patients with IRIS stage 3 and 4 disease treated with SUB placement for ureteral obstruction. Fungal infection is an important differential diagnosis to consider when ureteral obstruction occurs with acute kidney injury and grossly abnormal renal parenchyma.

\section{Ethics statement}

Consent was obtained from the client in order to include the patient in this case report. The best practice of veterinary care was undertaken for this patient. 


\section{Acknowledgment}

The authors thank Mara Hickey and Sherry Appel for their assistance with the manuscript.

\section{Disclosure}

The authors report no conflicts of interest in this work.

\section{References}

1. Berent AC. Ureteral obstructions in dogs and cats: a review of traditional and new interventional diagnostic and therapeutic options. JVet Emerg Crit Care. 2011;21(2):86-103.

2. Pressler BM, Vaden SL, Lane IF, Cowgill LD, Dye JA. Candida spp. urinary tract infections in 13 dogs and seven cats: predisposing factors, treatment, and outcome. J Am Anim Hosp Assoc. 2003;39(3):263-270.

3. Jin Y, Lin D. Fungal urinary tract infections in the dog and cat: a retrospective study (2001-2004). J Am Anim Hosp Assoc. 2005;41(6):373-381.

4. Lulich JP, Osborne CA. Fungal urinary tract infections. In: Kirk RW, Bonagura JD, editors. Current Veterinary Therapy XI: Small Animal Practice. Philadelphia, PA: Saunders; 1992:914-919.

5. Gerding PA, Morton LD, Dye JA. Ocular and disseminated candidiasis in an immunosuppressed cat. $\mathrm{J}$ Am Vet Med Assoc. 1994;204(10):1635-1638.

6. Coldrick O, Brannon CL, Kydd DM, Pierce-Roberts G, Borman AM, Torrance AG. Fungal pyelonephritis due to Cladophialophora bantiana in a cat. Vet Rec. 2007;161(21):724-728.

7. Tan RJ, Lim EW. Isolation of Torulopsis glabrata from a urine specimen of a Labrador bitch with urolithiasis. Br Vet J. 1977;133(3):324-325.

8. Kyles AE, Hardie EM, Wooden BG, et al. Clinical, clinicopathologic, radiographic, and ultrasonographic abnormalities in cats with ureteral calculi: 163 cases (1984-2002). J Am Vet Med Assoc. 2005;226(6):932-936.

9. Cannon AB, Westropp JL, Ruby AL, Kass PH. Evaluation of trends in urolith composition in cats: 5,230 cases (1985-2004). J Am Vet Med Assoc. 2007;231(4):570-576.

10. Kyles AE, Hardie EM, Wooden BG, et al. Management and outcome of cats with ureteral calculi: 153 cases (1984-2002). J Am Vet Med Assoc. 2005;226(6):937-944.

11. Nicoli S, Morello E, Martano M, Pisoni L, Buracco P. Double-J ureteral stenting in nine cats with ureteral obstruction. Vet J. 2012;194(1):60-65.
12. Horowitz C, Berent A, Weisse C, Langston C, Bagley D. Predictors of outcome for cats with ureteral obstructions after interventional management using ureteral stents or a subcutaneous ureteral bypass device. $J$ Feline Med Surg. 2013;15(12):1052-1062.

13. Berent AC, Weisse CW, Todd K, Bagley DH. Technical and clinical outcomes of ureteral stenting in cats with benign ureteral obstruction: 69 cases (2006-2010). J Am Vet Med Assoc. 2014;244(5):559-576.

14. Manassero M, Decambron A, Viateau V, et al. Indwelling double pigtail ureteral stent combined or not with surgery for feline ureterolithiasis: complications and outcome in 15 cases. J Feline Med Surg. 2014;16(8):623-630.

15. Cray M, Berent AC, Weisse CW, Bagley D. Treatment of pyonephrosis with a subcutaneous ureteral bypass device in four cats. J Am Vet Med Assoc. 2018;252(6):744-753.

16. Pressler BM. Candidiasis and rhodotorulosis. In: Greene CE, editor. Infectious Diseases of the Dog and Cat. 4th ed. St. Louis, MO: Elsevier/ Saunders; 2012:666-672.

17. de Pauw B, Walsh TJ, Donnelly JP, et al. Revised definitions of invasive fungal disease from the European Organization for Research and Treatment of Cancer/Invasive Fungal Infections Cooperative Group and the National Institute of Allergy and Infectious Diseases Mycoses Study Group (EORTC/MSG) Consensus Group. Clin Infect Dis. 2008;46(12):1813-1821.

18. Ascioglu S, Rex JH, de Pauw B, et al. Defining opportunistic invasive fungal infections in immunocompromised patients with cancer and hematopoietic stem cell transplants: an international consensus. Clin Infect Dis. 2002;34(1):7-14.

19. Gomez CA, Budvytiene I, Zemek AJ, Banaei N. Performance of targeted fungal sequencing for culture-independent diagnosis of invasive fungal disease. Clin Infect Dis. 2017;65(12):2035-2041.

20. Roberts SF, Aronson LR, Brown DC. Postoperative mortality in cats after ureterolithotomy. Vet Surg. 2011;40(4):438-443.

21. Wormser C, Clarke DL, Aronson LR. Outcomes of ureteral surgery and ureteral stenting in cats: 117 cases (2006-2014). J Am Vet Med Assoc. 2016;248(5):518-525.

22. Culp WT, Palm CA, Hsueh C, et al. Outcome in cats with benign ureteral obstructions treated by means of ureteral stenting versus ureterotomy. $J$ Am Vet Med Assoc. 2016;249(11):1292-1300.

23. Weese JS, Blondeau JM, Boothe D, et al. Antimicrobial use guidelines for treatment of urinary tract disease in dogs and cats: Antimicrobial guidelines working group of the international society for companion animal infectious diseases. Vet Med Int. 2011;2011:263768.
Veterinary Medicine: Research and Reports

\section{Publish your work in this journal}

Veterinary Medicine: Research and Reports is an international, peer-reviewed, open access journal publishing original research, case reports, editorials, reviews and commentaries on all areas of veterinary medicine. The manuscript management system is completely online and includes a very quick and fair peer-review system.

\section{Dovepress}

Visit http://www.dovepress.com/testimonials.php to read real quotes from published authors. 